\title{
Influence of residual stresses and geometric imperfections on the elastoplastic collapse of cylindrical tubes under external pressure
}

\author{
Philippe Le Grognec ${ }^{\mathrm{a},{ }^{*}}$, Pascal Casari ${ }^{\mathrm{b},{ }^{*}}$ and Dominique Choqueuse ${ }^{\mathrm{c}, \text { * }}$
}

\footnotetext{
${ }^{a}$ Ecole des Mines de Douai, Polymers and Composites Technology \& Mechanical Engineering Department, 941 rue Charles Bourseul - BP 10838, 59508 Douai Cedex, France

${ }^{\mathrm{b}}$ GeM (Laboratory of Civil and Mechanical Engineering), Institut Universitaire de Technologie de Saint-Nazaire, 58 rue Michel-Ange - BP 420, 44606 Saint-Nazaire Cedex, France

${ }^{c}$ Ifremer, Materials and Structures Department, Technopôle de Brest-Iroise - BP 70, 29280 Plouzané, France
}

*: Corresponding author: Philippe Le Grognec, Tel.: +33 (0)3 277123 21; fax: +33 (0)3 27712981 , email address : legrognec@ensm-douai.fr, Pascal Casari : pascal.casari@univ-nantes.fr, Dominique Choqueuse : Dominique.Choqueuse@ifremer.fr

\begin{abstract}
:
The buckling problem of a circular cylindrical shell has long been widely investigated due to its great importance in the design of aerospace and marine structures. Geometric imperfections and residual stresses are inevitable in practice and have been so far frequently considered in analytical and numerical predictions. But little attention has been paid until now on the combined influence of such initial defects on the critical and often unstable response of such elastoplastic structures. In this paper, a shell finite element is designed within the total Lagrangian formulation framework to deal with the elastoplastic buckling and post-buckling of thin cylindrical tubes under external pressure and axial compression. A specific experimental process will be introduced in order to measure residual stresses in the shell very accurately, so as to include them in the numerical calculations. The present formulation will enable us to describe the complete non-linear solutions, namely the critical pressures (bifurcation and limit (collapse) loads), the bifurcation modes and the bifurcated equilibrium branches up to advanced post-critical states. Comparisons will be made between numerical results and the experimental critical value and deformation patterns of a new generation profiler. Furthermore, the combined effects of geometric imperfections, residual stresses and plasticity will be analyzed.
\end{abstract}

Keywords: Buckling; Elastoplasticity; Imperfection sensitivity; Residual stresses; Cylindrical containers under external pressure 


\section{Introduction}

Failure of thin structures submitted to compressive loadings is mainly due to the buckling phenomenon. Their mechanical design implies thus the analysis of their buckling and post-buckling behaviors, namely the calculation of the critical loads, the bifurcation modes and possibly the post-critical equilibrium branches.

In elasticity, the bifurcation is related to the structural instability as shown in Koiter's theory. The problem is much more difficult with thicker structures where plasticity may occur before buckling. From a theoretical point of view, Shanley (1947) was the first to give the tangent modulus critical load for a discrete model. Then, Hill (1958) extended these results to a three-dimensional continuum by using the concept of "comparison elastic solid". He examined the uniqueness and stability criteria, and pointed out the difference between bifurcation and stability.

A comprehensive work on the buckling of structures was presented by Brush and Almroth (1975). They examined the effect of initial imperfections, inherent in many real structures, on the critical loads. When dealing with the buckling phenomenon, cylindrical shells are certainly among the most sensitive structures to all imperfection types (in comparison with beams and plates) and, as a matter of fact, very useful in industrial applications. For example, the bifurcation of a cylinder under axial compression, like a spherical shell under external pressure, corresponds to a sub-critical point (a load limit point on the bifurcated branch), namely an upper bound for the strength of the shell in the post-critical behavior. For that reason, the critical problem of a cylinder under axial compression was the subject of a lot of experimental and numerical investigations (Bushnell, 1982, 1985). A wide variety of experimental results were obtained in terms of the boundary conditions, the geometries and the material parameters. Furthermore, different numerical models were devised in order to predict the critical loads as well as the advanced post-buckling behavior. Among others, Tvergaard (1983) analyzed the effect of geometric and material parameters on the buckling behavior of a cylinder under axial compression. It was also shown that the boundary conditions are a deciding factor for obtaining various types of buckling modes. From a material point of view, different critical values are obtained in the literature, depending on the plasticity theory considered. In the case of a cylinder under axial compression, the critical stresses obtained by the deformation theory are fairly far from the ones corresponding to the flow theory. As a matter of fact, the

\footnotetext{
* Corresponding author.

Email addresses: legrognec@ensm-douai.fr (Philippe Le Grognec), pascal.casari@univ-nantes.fr (Pascal Casari), dominique.choqueuse@ifremer.fr (Dominique Choqueuse).
} 
solutions derived from the deformation theory turn out to compare best with the experimental results, although such a theory does not take into account the elastic unloading possibility. On the contrary, the flow theory overpredicts the critical loads. Comparisons between the predictions of both flow and deformation theories and experimental results were recently performed in Bardi and Kyriakides (2006) and Bardi, Kyriakides and Yun (2006), in the case of axially loaded cylindrical shells, including the effect of anisotropy with the use of Hill's yield criterion. The flow theory is shown to significantly overpredict both the critical stresses and strains, whereas the deformation theory gives rise to predictions much closer to the experimental values.

For this loading case, large discrepancies have always been observed between numerical and experimental results. The most significant feature is that the experimental critical loads sometimes fall down to less than half of the numerical values, see for instance Singer (1999) for a review on the importance of shell buckling experiments. These differences, due to unavoidable imperfections in experiments, were explained by many authors. If the major influence of geometric imperfections on the buckling behavior of cylindrical shells is universally recognized, the importance of residual stresses has also been enhanced in the case of axial compressive stresses. Just let us mention Mandal and Calladine (2000) and Lancaster, Calladine and Palmer (2000), who investigated the buckling under self-weight of cylinders with free ends by means of an adequate experimental set-up. Since the ends were able to move freely in the axial direction, there were no longer imperfections due to localized stresses and the critical value was found to increase in comparison to the classical experimental result obtained with enforced displacements at the ends. Therefore, these authors concluded that localized stresses were strongly responsible for the drop of the critical value.

In some cases, the structure may be quite insensitive to imperfections in elasticity and yet markedly sensitive in plasticity. Cylindrical shells under external pressure are quite less imperfection-sensitive than axially compressed ones but their sensitivity is more noticeable in the plastic domain than in elasticity. As a matter of fact, this problem has more rarely been studied in the literature. Let us mention the buckling of offshore pipelines under external pressure as one of the main application exhaustively studied by Kyriakides and Corona (2007). These authors examined the occurence of elastic or plastic buckling/collapse depending on the thinness of the tubes and also the influence of several types of imperfections on the critical loads. The combination of bending and external pressure had previously been addressed from both experimental and numerical points of view by the same authors (Corona and Kyriakides, 1988). They came to the conclusion that practical factors that mainly affect the buckling of cylindrical shells under external pressure are geometric imperfections (initial ovality or thickness variations), residual stresses and yield anisotropy. Other discrepancies between experimental and simulation results could also 
come from the experimental imperfect boundary conditions or loadings; they could finally result from material heterogeneities, which are often difficult to be measured.

In elasticity, just few authors investigated the imperfection sensitivity of a cylinder under external pressure. For example, Gusic, Combescure and Jullien (2000) analyzed the influence of thickness imperfections on the buckling behavior. But in many practical applications, the cylindrical shell may buckle plastically, or at least, collapse plastically as soon as plasticity occurs in the post-buckling stage. In this case, one deals with a limit load instability due to interactions between geometric and material non-linearities, which leads to a more or less localized collapse (Dyau and Kyriakides, 1993).

In that context, many studies deal with the unstable collapse of cylinders, considering all sorts of imperfections. Until now, most attention has been paid to geometric imperfections. The effect of thickness variations on plastic buckling and collapse of pipelines under external pressure was analyzed in Yeh and Kyriakides (1986) and Yeh and Kyriakides (1988), where it was shown that this type of geometric imperfection, as well as wall eccentricity, has a secondary influence on the critical behavior, compared with an initial ovality, as long as realistic amplitudes are considered.

Imperfections in the form of residual stresses have more rarely been investigated. Most of the time, the residual stresses just enhance the yielding plastic zone in the structure and therefore decrease the buckling load. In elastoplastic column buckling problems, for which residual stresses are important and widely recognized, initial stresses have never shown any effect on the buckling loads, as far as an homogeneous purely elastic or plastic behavior is assumed. The effect of residual stresses is thus mostly significant for members of mid-slenderness, when non-homogeneous (partly elastic and plastic) prebifurcation conditions are involved (see Triantafyllidis (1983) for an analysis of general non-homogeneous pre-bifurcation conditions). This property is due to the simple residual stress patterns and the one-dimensional stress state encountered in such wire structures, and is no longer valid for plates or shells. The effect of residual stresses on the buckling of shell-type structures has recently been examined by a few authors. The problem of collapse under combined bending and external pressure was revisited by Corona and Kyriakides (2000) in order to take into account asymmetric imperfections together with residual stresses and better explain the scatter between experimental and numerical solutions under high pressures. See also Kyriakides and Corona (2007) for a complete review on the subject of elastoplastic buckling and collapse of cylindrical shells under various loads (among which external pressure) with geometric and material imperfections, including residual stresses.

Analytical solutions can be derived for the critical loads and bifurcation modes 
of beams, plates or possibly shells with simple boundary conditions and loadings, under homogeneous pre-bifurcation conditions or even in the presence of simple initial imperfections, both in elasticity and plasticity (see the pioneering works of Timoshenko and Gere (1961) in elasticity and Dubey (1969) in plasticity, for instance). Intermediate semi-analytical approaches involving asymptotic expansions can be derived in order to estimate not only the buckling behavior but also the post-bifurcated solution. For example, in his asymptotic analysis of plastic post-buckling, Hutchinson (1974) examined the influence of various geometric imperfection types on the buckling and postcritical behavior of several structures, among which cylindrical shells under various loads. It allowed him to determine safety factors for the critical load versus the imperfection size and particularly to assess the stability of the bifurcated branch. Likewise, in order to include a non-linear pre-buckling behavior, Kheyrkhahan and Peek (1999) proposed an asymptotic analysis using a Lyapunov-Schmidt-Koiter decomposition and an asymptotic expansion technique in the framework of the finite element method. But as far as the complete non-linear solution is required or complex initial conditions are assumed, exact solutions obtained by numerically tracking the bifurcated equilibrium branches and giving rise, if needed, to advanced post-critical states are preferred. In the case of compressed shells such as cylinders, which are usually very imperfection-sensitive, numerical tools are thus most often employed to analyze their buckling behavior and collapse.

The present work deals with the buckling behavior and collapse of cylindrical tubes submitted to both hydrostatic external pressure and axial compression (end effect) in seabeds. Some experiments performed by one of the authors seem to be in disagreement with classical linearized elastic buckling pressures. All the above-mentioned facts show that it is preferable to use a numerical tool to analyze the influence of geometric imperfections and residual stresses, among others, on the complete non-linear buckling and post-buckling response of such compressed shells. It will be shown that the occurrence of plasticity is mainly responsible for the early collapse of the shell. Yielding is caused by the combination of stresses induced by the applied loads with the residual stresses which remain after the manufacturing process, so that residual stresses are of great importance. Moreover, it will be shown that it is difficult to separate the effects of residual stresses and geometric imperfections, such as the ovality of the initially perfect circular cylindrical shell.

\section{Outline of the paper}

This paper is devoted to the understanding of the buckling and post-buckling behaviors of a cylindrical tube under combined external pressure and axial compression in the elastoplastic range, taking into consideration geometric 
imperfections together with residual stresses. A specific numerical tool has been developed to perform the numerical computation of the critical loads, the bifurcation modes and the advanced post-critical states.

The virtual work principle and the total Lagrangian formulation are used to build a shell finite element encompassing geometric non-linearities (finite rotations) and material non-linearities (elastoplastic behavior with the von Mises yield criterion and a linear isotropic hardening). We shall only focus on the physically more correct flow plasticity theory, observing that both flow and deformation theories of plasticity give rise to nearly similar results in the case of paramount external pressure. The shell element is designed to deal with buckling and advanced post-buckling of thin structures. A specific arc-length method and branch-switching procedures are included in order to resolve snapthrough (or even snap-back) and bifurcation phenomena, encountered when dealing with cylindrical shells under external pressure.

First, the numerical formulation will be concisely presented. Then a specific experimental process will be introduced in order to measure residual stresses in the shell very accurately. Numerical results will finally be presented, relating to a new generation profiler, emphasizing the relative and total effect of residual stresses and geometric imperfections on the elastic or plastic buckling loads and collapse behavior. The finite element formulation and the solution procedure will be validated by comparison with related experimental data.

\section{Shell finite element formulation}

The elastoplastic thin shell finite element formulation is briefly reviewed in the following paragraphs. A more detailed description, which is outside the scope of this paper, may be found in Le Grognec and Le van (2008).

\subsection{Shell kinematics with finite rotations}

The shell-like body is represented by a middle surface and an inextensible director field (in the direction of the shell thickness) which is not constrained to be normal to the middle surface, except at the reference configuration, thus following a Mindlin-Reissner type formulation.

The motion of the inextensible director is described by:

$$
\vec{t}=\mathbf{R} \cdot \vec{T}
$$


where the rotation tensor $\mathbf{R}$ is orthogonal $\left(\mathbf{R}^{-1}=\mathbf{R}^{T}\right)$ and can be associated with the skew-symmetric tensor $\Theta$ through the exponential mapping:

$$
\mathbf{R}=\exp \Theta=\sum_{k=0}^{+\infty} \frac{\Theta^{k}}{k !}
$$

Let us define the axial vector $\vec{\theta}$ by $\Theta . \vec{v}=\vec{\theta} \wedge \vec{v}, \forall \vec{v} \in \mathbb{R}^{3}$. From the equality $\mathbf{R} . \vec{\theta}=\vec{\theta}$, it follows that $\mathbf{R}$ is the rotation about $\vec{\theta}$. Then an explicit expression for Equation (2) is given by the Rodrigues formula:

$$
\mathbf{R}=\cos \theta \mathbf{I}+\frac{\sin \theta}{\theta} \Theta+\frac{1-\cos \theta}{\theta^{2}} \vec{\theta} \otimes \vec{\theta}
$$

where $\theta=\|\vec{\theta}\|$ is the magnitude of the rotation.

Since $\vec{\theta}$ is perpendicular to $\vec{T}$, the director field $\vec{t}$ can be recast in the simplified form:

$$
\vec{t}=\mathbf{R} \cdot \vec{T}=\cos \theta \vec{T}+\frac{\sin \theta}{\theta} \vec{\theta} \wedge \vec{T}
$$

\subsection{Elastoplasticity formulation}

The equation set for finite elastoplasticity is established on the basis of the total Lagrangian formulation and the generalized standard materials theory introduced by Halphen and Nguyen (1975). The elastic strain is related to the second Piola-Kirchhoff stress by the three-dimensional Saint-Venant-Kirchhoff law, including the residual stress state at the reference configuration. Since the normal material stress is assumed to be zero, the elastic fourth-order tensor $\mathbf{D}$ must be replaced with a reduced one $\mathbf{C}$ for the shell model. Following Green and Naghdi (1965), the Green strain tensor $\mathbf{E}$ is split additively into its elastic and plastic parts. Use is made of the von Mises yield criterion with a linear isotropic hardening.

\subsection{The discretized non-linear equations}

Use is made of the virtual work principle in order to obtain the discretized nonlinear equations. The middle surface is discretized using eight-node isoparametric shell finite elements. There are five degrees of freedom at each node: three translations and two rotations in the tangent space. The reference position, the displacement on the middle surface, and the director vectors $\vec{T}$ and $\vec{t}$ are interpolated in the same way. 
The integration over the middle surface and through the thickness are performed using the Gaussian quadrature. Eventually, the virtual work principle yields the non-linear discretized equation system:

$$
\{\mathbf{R}(\mathbf{U})\}=\{\Psi\}-\{\Phi\}=\{0\}
$$

where $\{\mathbf{U}\}$ denotes the nodal displacement vector of the whole structure, $\{\mathbf{R}\}$ the residual vector, $\{\Psi\}$ and $\{\Phi\}$ are the internal and external force vectors, respectively.

\section{Solution procedure}

\subsection{Local integration}

The non-linear equation system (5) is solved by the iterative Newton-Raphson procedure, which requires the computation of the structural tangent stiffness matrix:

$$
\left[\mathbf{K}_{T}\right]=\frac{\partial\{\mathbf{R}(\mathbf{U})\}}{\partial\{\mathbf{U}\}}
$$

At each iteration, the local integration consists in solving the following problem: assuming the strain $\mathbf{E}$ is known, compute the plastic strain $\mathbf{E}^{p}$, the stress $\Sigma$ and the reduced consistent elastoplastic tangent modulus $\mathbf{C}^{p}$. The implicit Euler scheme and an algorithm of radial return type are employed to integrate the state and evolution laws as described in Simo and Hughes (1998).

\subsection{Arc-length method}

The nodal displacement vector is split into two parts: one denoted by $\{\widetilde{\mathbf{U}}\}$ contains the unknown degrees of freedom, the other denoted by $\{\overline{\mathbf{U}}\}$ contains the prescribed degrees of freedom. The external force and the tangent stiffness matrix are split in a similar way:

$$
\{\mathbf{U}\}=\left\{\begin{array}{l}
\widetilde{\mathbf{U}} \\
\overline{\mathbf{U}}
\end{array}\right\} \quad\{\Phi\}=\left\{\begin{array}{l}
\widetilde{\Phi} \\
\bar{\Phi}
\end{array}\right\} \quad\left[\mathbf{K}_{T}\right]=\left[\begin{array}{ll}
\widetilde{\widetilde{K}}_{T} & \widetilde{\mathbf{K}}_{T} \\
\widetilde{\mathbf{K}}_{T} & \overline{\mathbf{K}}_{T}
\end{array}\right]
$$

At each increment, the quadratic arc-length method is used in order to proceed on the equilibrium branches given a specified arc-length $\Delta l$, see Riks (1979) 
and Crisfield (1991). The constraint equation writes, assuming a proportional prescribed loading:

$$
\|\Delta \widetilde{\mathbf{U}}\|^{2}+\Delta \lambda^{2} C_{r e f}^{2}=\Delta l^{2}
$$

where $\lambda$ represents the load factor and $C_{r e f}$ is a scale parameter which makes the relation consistent dimensionally.

Combining Equation (8) with the equilibrium equation leads to a quadratic equation. When solving this quadratic equation, one may encounter severe computational difficulties due to complex roots which occur repeatedly. It is found that an efficient way to cope with these complex roots is to modify the standard solution scheme according to Lam and Morley (1992). The main idea is to project the residual force onto the external load vector. At a current iteration where complex roots occur, the residual force is split into one component in the load direction and another component orthogonal to this load. The last component is mainly responsible for the complex roots and should be eliminated.

\subsection{Branching method}

Branch-switching techniques are included in the numerical procedure in order to detect the bifurcation points and bifurcate onto a given branch. Specific methods are implemented following Riks (Riks, 1979, 1991) and Seydel (1994). The four fundamental steps are given below.

1. At the end of each increment, it must be checked whether one has gone across one or several critical points. The detection of critical points is based on the singularity of the tangent stiffness matrix, which is factorized following the Crout formula. The critical points are determined by counting the negative pivot number.

2. Each critical point detected has to be isolated in order to determine its nature: limit point or bifurcation point. To do this, the current arc-length $\Delta l$ is re-estimated several times using a dichotomy-like method. A simple way to distinguish a limit point from a bifurcation point is to determine the sign of the current stiffness parameter introduced by Bergan, Horrigmoe, Krakeland and Soreide (1978):

$$
k=\frac{\left\{\widetilde{\Phi}_{r e f}\right\}^{T}\left\{\widetilde{\mathbf{U}}_{\Phi r e f}\right\}}{\left\{\widetilde{\mathbf{U}}_{\Phi r e f}\right\}^{T}\left\{\widetilde{\mathbf{U}}_{\Phi r e f}\right\}}
$$


where $\left\{\widetilde{\Phi}_{r e f}\right\}$ is the reference force vector and $\left\{\widetilde{\mathbf{U}}_{\Phi r e f}\right\}=\left[\widetilde{\mathbf{K}}_{T}\right]^{-1}\left\{\widetilde{\Phi}_{r e f}\right\}$. The sign of parameter $k$ changes when passing a limit point, whereas it remains unchanged when passing a bifurcation point.

3. If it is a bifurcation point, the step increment is renewed so as to reach a point just behind the bifurcation point.

4. Finally, the switching on a bifurcated branch is performed using the mode injection method (Wagner and Wriggers, 1988; Seydel, 1994): at the first step of a bifurcating branch, the eigenvector $\{\widetilde{\mathbf{Z}}\}$, solution of $\left[\widetilde{\mathbf{K}}_{T}\right]\{\widetilde{\mathbf{Z}}\}=\{\mathbf{0}\}$, is computed and the following predictions are used:

$$
\delta \lambda=0 \quad\{\delta \widetilde{\mathbf{U}}\}= \pm \frac{\{\widetilde{\mathbf{Z}}\}}{\|\widetilde{\mathbf{Z}}\|}
$$

\section{Experimental data}

\subsection{Context}

Unexpected collapse of cylindrical aluminum shells subjected to hydrostatic compression has been recently observed during qualification of equipments (Ifremer). These cylindrical shells were designed as electronic containers for subsurface Lagrangian drift buoys used in worldwide program of ocean monitoring (Coriolis). Analytical models are used to design the containers. These models were previously validated by experimental tests on structures and finite element modeling in the framework of linearized elastic buckling, and were supposed to guarantee a conservative design.

After analysis, the unexpected collapse was attributed to the presence of initial geometric imperfections, but also non-negligible residual stresses which were not taken into account in the models, induced during the manufacturing process (aluminum T6 instead of T6511 grade), and then to an overestimation of the capacity of the container to withstand the pressure. Indeed, a new grade of aluminum has been selected for a new application which can only be provided in T6 grade, so that residual stresses are still present in the material and their effect on the capability of the structures to withstand the pressure must be evaluated.

The following section aims at estimating the influence of such residual stresses, in addition to plasticity and geometric imperfections, on the collapse behavior 
of one cylindrical container, using a complete non-linear buckling and postbuckling analysis based on the formulation presented above.

\subsection{Geometric and material data}

Let us consider a cylindrical tube defined in the reference configuration by the length $l$, the average radius $R$ and the thickness $h \ll R$. The shell is made of an elastoplastic material and a linear isotropic hardening is assumed (what can properly stand for the real stress-strain curve). The numerical values are listed in Tables 1 and 2.

Table 1

\begin{tabular}{|c|c|c|}
\hline Length $l$ & Radius $R$ & Thickness $h$ \\
\hline $970 \mathrm{~mm}$ & $53.375 \mathrm{~mm}$ & $6.75 \mathrm{~mm}$ \\
\hline
\end{tabular}

Geometric parameters

Table 2

\begin{tabular}{|c|c|c|c|}
\hline $\begin{array}{c}\text { Young's } \\
\text { modulus } E\end{array}$ & $\begin{array}{c}\text { Poisson's } \\
\text { ratio } \nu\end{array}$ & $\begin{array}{c}\text { Yield stress } \\
\sigma_{0}\end{array}$ & $\begin{array}{c}\text { Isotropic hardening } \\
\text { modulus } H\end{array}$ \\
\hline $71000 \mathrm{MPa}$ & 0.32 & $365 \mathrm{MPa}$ & $625 \mathrm{MPa}$ \\
\hline
\end{tabular}

Material properties

This cylindrical tube is submitted to a uniform hydrostatic external pressure $P$, and axial compression due to the head and bottom of the tube, whose geometry will not be modeled. The ends of the cylinder are clamped for the same reason.

\subsection{Collapse experiment}

The cylindrical shell has been equipped with strain gauges, as seen in Figure 1, in order to record the local deformations of the structure during the pressure loading.

A set of six biaxial strain gauges (axial and circumferential) has been bonded on the structure to cover the whole external circumference of the central part of the tube. The position of the gauges is described in Figure 2. This configuration has been chosen taking into account the expected mode shape of the structure.

End closures are fixed at the ends of the tube in order to ensure a perfect watertightness and the structure is tested in a hyperbaric tank. A speed of $1 \mathrm{MPa} / \mathrm{min}$ for pressure increase has been retained, following the loading history depicted in Figure 3. 


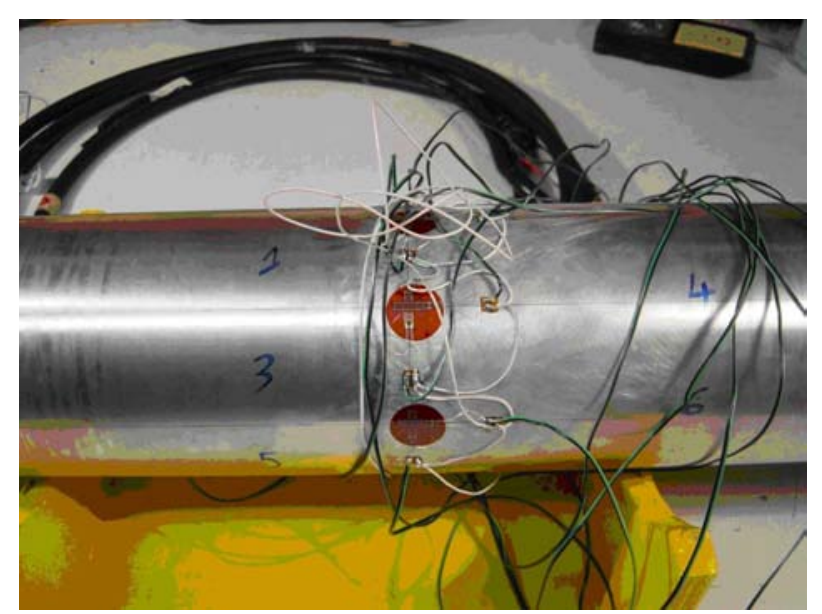

Fig. 1. Strain gauges

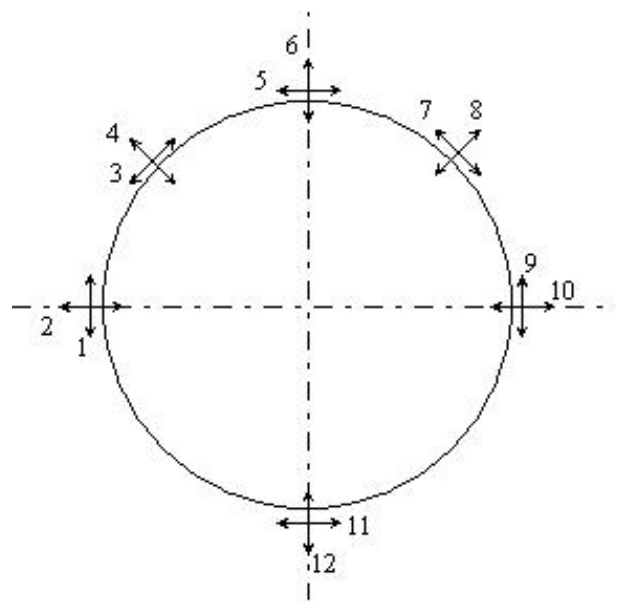

Fig. 2. Circumferential position of the strain gauges

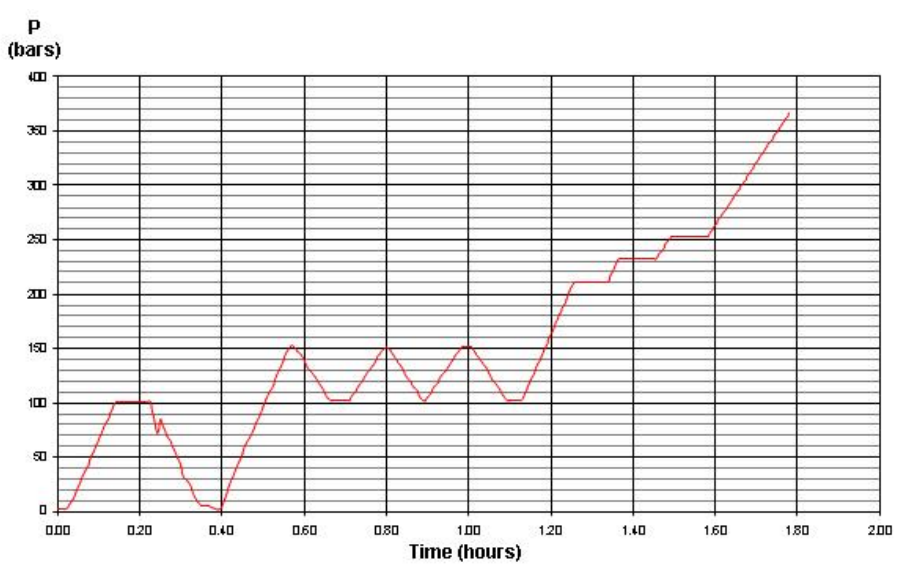

Fig. 3. Applied external pressure during the experimental test

The complete experimental set-up is presented in Figure 4. The circumferential microstrains are recorded during the test and are reported in Figure 5. The failure of the structure occurred at $P=366$ bars by unstable buckling, as 
shown in Figure 6.

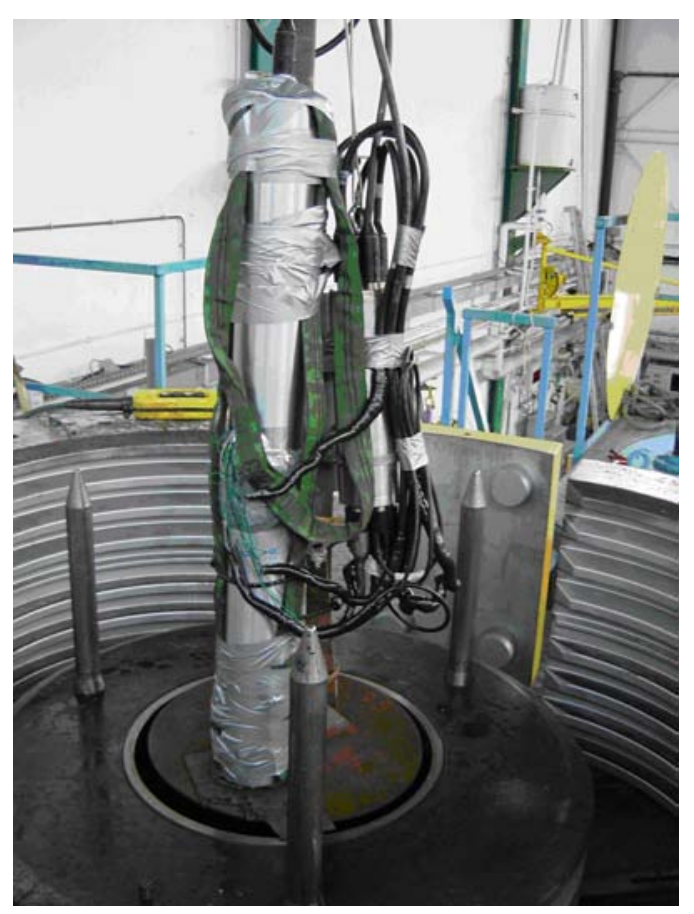

Fig. 4. Cylindrical tube at the initial state



Fig. 5. Circumferential microstrains around the tube vs. applied external pressure A linear response of the strain gauges is observed until about 300 bars, where divergence between the different sensors seems to appear. In order to highlight this divergence phenomenon, the following expression could be proposed as an indicator of the "ovalization" of the initially circular central circumference:

$$
\begin{aligned}
\varepsilon_{\text {oval }}= & \varepsilon_{\text {gauge }}-\frac{1}{2}\left(\operatorname{Min}\left(\varepsilon_{J 1}, \varepsilon_{J 3}, \varepsilon_{J 5}, \varepsilon_{J 7}, \varepsilon_{J 9}, \varepsilon_{J 11}\right)\right. \\
& \left.+\operatorname{Max}\left(\varepsilon_{J 1}, \varepsilon_{J 3}, \varepsilon_{J 5}, \varepsilon_{J 7}, \varepsilon_{J 9}, \varepsilon_{J 11}\right)\right)
\end{aligned}
$$

Figure 7 indicates that the gauges placed at $0^{\circ}$ and $180^{\circ}$ display values of $\varepsilon_{\text {oval }}$ 


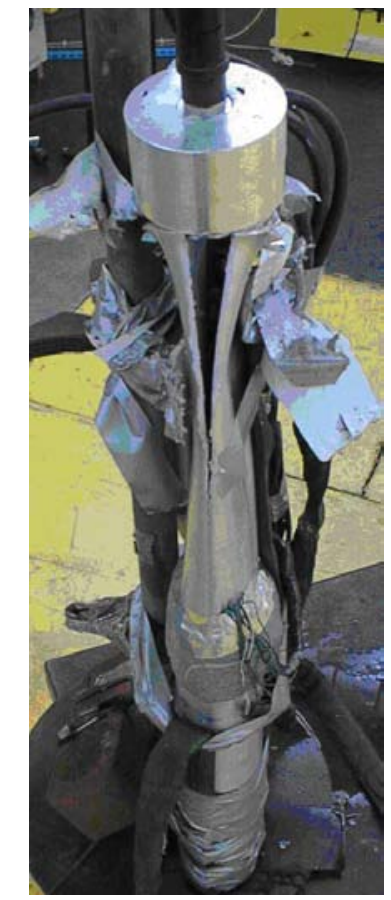

Fig. 6. Collapse of the cylindrical tube

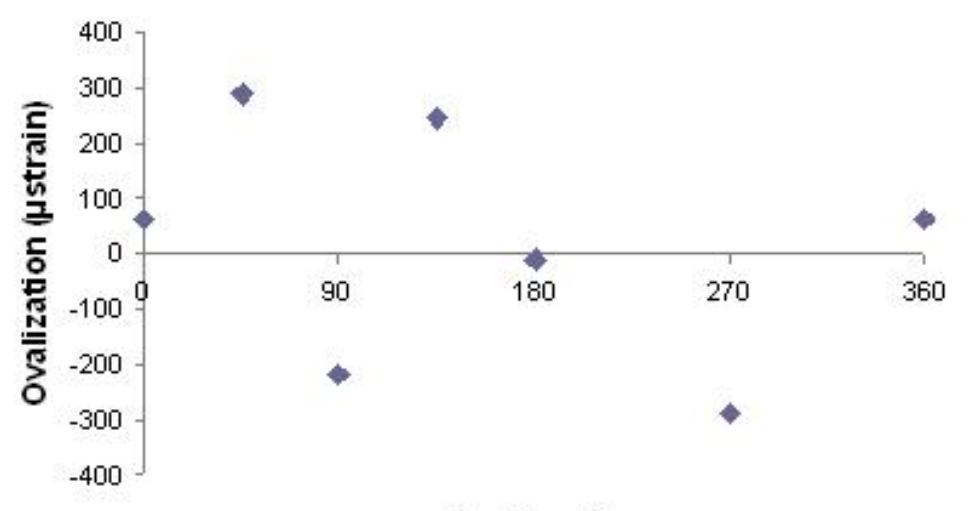

Position $\left({ }^{\circ}\right)$

Fig. 7. Ovalization indicator around the circumference at a high pressure level

very close to zero and thus are located on nodes of ovalization. Gauges J3, J5, $\mathrm{J} 7$ and $\mathrm{J} 9$, placed respectively at $45^{\circ}, 90^{\circ}, 135^{\circ}$ and $270^{\circ}$, have a significant $\varepsilon_{\text {oval }}$ and can be used to reveal the ovalization process. As an example, in Figure 8 are plotted the ovalization parameters for J3 and J5 during the whole test. These results clearly indicate the divergence of the microstrains around $200-250$ bars, long before the buckling phenomenon. Therefore, an initial ovality of the shell should be introduced in the geometry, in order to better describe the collapse of the tube, which probably depends on this initial geometric imperfection. 


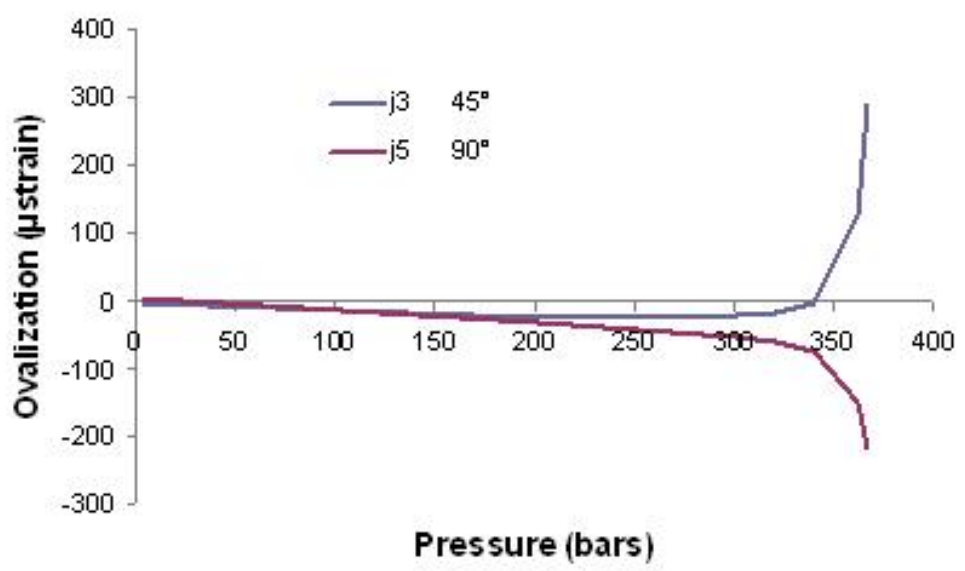

Fig. 8. Ovalization growth

\subsection{Measurement of residual stresses}

The main objective of the following section is to better evaluate numerically the collapse load of the containers, taking into account initial geometric imperfections but also initial stresses. Indeed, the manufacturing process of such aluminum tubes involves both axial and circumferential non-negligible residual stresses related to the cycle of temperature submitted to the material during manufacture. Therefore, accurate measurements of these initial stress fields are needed to complete the model data.

\subsubsection{Experimental procedure}

The residual stresses are assumed to depend on the radial position only. Axial and circumferential gauges are bonded on the inner wall of the tube. Then, stresses are progressively released, using chip removal machining (turning) on the outer wall surface. Machining parameters are principally the depth of cut $(0.5 \mathrm{~mm})$ and the sampling frequency $(2 \mathrm{~Hz})$. The response curves (longitudinal and circumferential strains during the turning process) are presented in Figure 9. Each zone with an oscillatory behavior corresponds to a material removal.

\subsubsection{Data reduction}

Some specific assumptions must be made in order to derive the internal stresses from the strains plotted in Figure 9. First, the plane stress condition is assumed, due to the thinness of the tube. Stresses are supposed to be piecewise constant (one value for each layer). During deformation, the tube remains cylindrical: its diameter and radius are changing but there is neither ovaliza- 




Fig. 9. Evolution of the longitudinal and circumferential strains during the turning process

tion nor local or global bending curvature in the tube.

Considering all the hypotheses above, the stresses in the tube are simply derived from the corresponding strains using the classical linear elastic Hooke's law in small strains. Then, the stresses released on the opposite side are deduced, following the iterative procedure illustrated by Figure 10 and explained below.

Once a layer of material is removed, stresses are released that give rise to particular strains on the opposite wall, where gauges are located. It allows one to calculate the variation of stresses in the remaining part of the cylinder and the amplitude of the released stresses coming from the layer removed (see Figure 10b).

Before a second layer is removed, the internal equilibrium is achieved, ensuring consistent residual stresses in the thickness (see Figure 10c).

Removing a second layer gives rise to a new stress release related to the deformation history. With such a sequential proceeding, the longitudinal and circumferential stresses are iteratively evaluated in each successive layer, and then smoothed to make easier the numerical implementation (Figures 11 and 12).

Jiao and Zhao (2003) also measured the through-thickness variation of residual stresses in circular tubes manufactured by several methods. They always found similar results, namely compressive stresses on the inside surface and tensile ones on the outside surface, for both longitudinal and transverse directions. Moreover, the residual stress patterns obtained experimentally (especially the small decrease at both internal and external radii) are very likely for such extruded tubes that are currently straightened afterwards. 


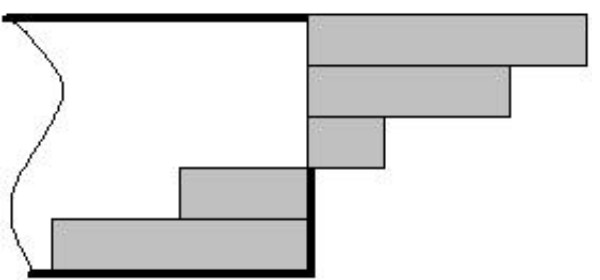

(a) Initial state

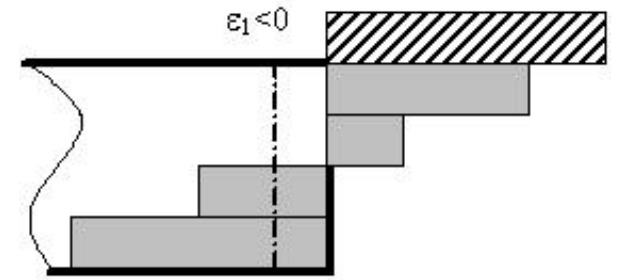

(b) Deformed state with one layer less

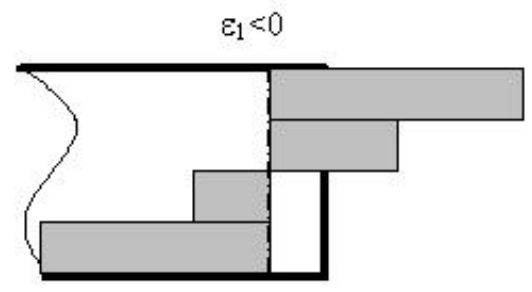

(c) New state of equilibrium

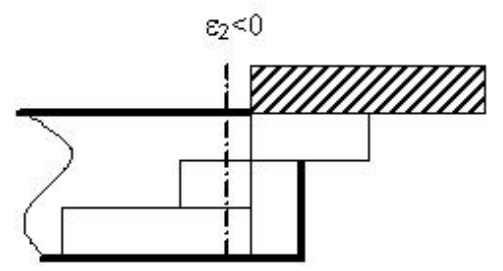

(d) Deformed state with two layers less

Fig. 10. Iterative procedure for the calculation of residual stresses

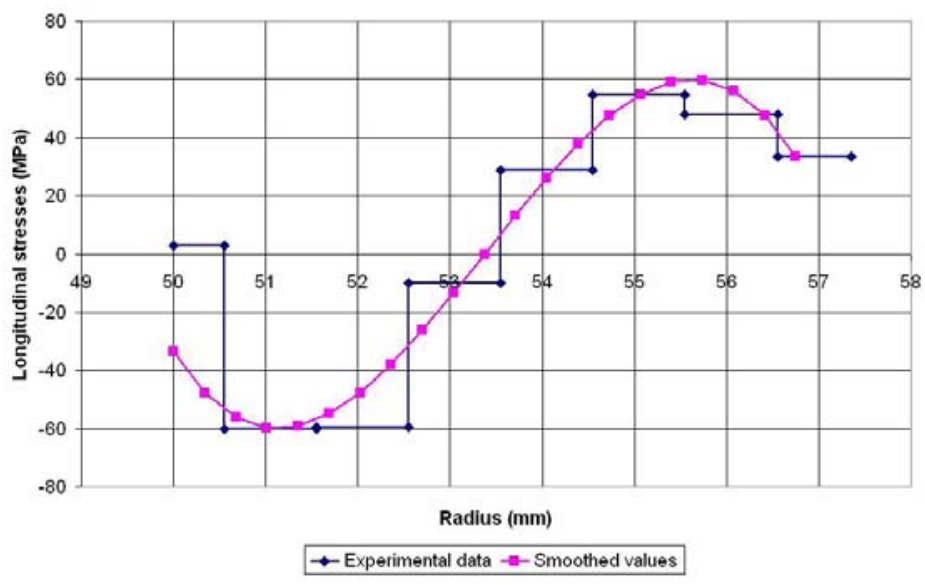

Fig. 11. Variation of longitudinal residual stresses in the shell thickness

At this stage, it can be mentioned that the maximum amplitude of both axial and circumferential residual stresses is large enough to modify significantly the carrying capacity of the tube. The most harmful stress values are the compressive ones, which are located on the inside surface and will be added to the compressive stresses due to the external pressure. 


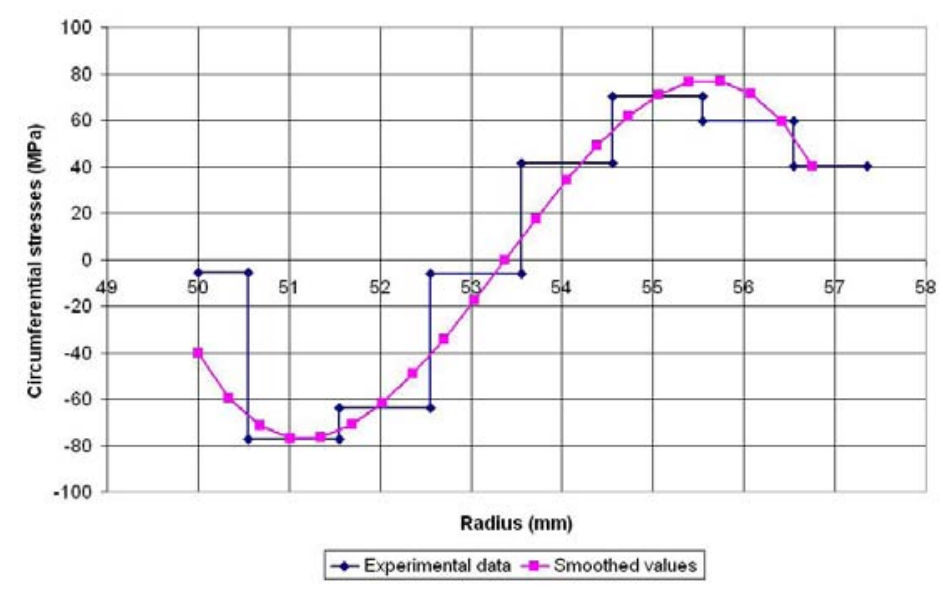

Fig. 12. Variation of circumferential residual stresses in the shell thickness

\section{Numerical results: validation and analysis}

\subsection{Numerical calculations}

In the following calculations, symmetries are taken into account in such a way that only one eighth of the cylinder is meshed, what considerably reduces the computational cost.

A few preliminary mesh convergence tests lead us to use about 200 quadratic elements for the considered portion of the cylinder and 4 or 7 Gaussian points for the numerical quadrature through the shell thickness, depending whether the behavior is assumed to be elastic or elastoplastic.

\subsubsection{Perfect cylindrical shell}

A first calculation is performed, considering a perfectly circular cylindrical shell without any residual stresses.

In elasticity, a bifurcation point is obtained at the critical pressure $P_{c}=$ 39.55 MPa. This bifurcation point is super-critical, namely the pressure increases in the post-buckling range. It corroborates the little imperfection sensitivity of a cylindrical shell under external pressure in elasticity. Indeed, the axial compression has very little effect on the buckling load and the global behavior is governed by the external pressure. As a consequence, no limit load has been found and no unstable collapse is expected.

When plasticity is considered, the critical pressure remains identical. Indeed, plasticity may occur at $P_{0}=\frac{2 h \sigma_{0}}{\sqrt{3} R}=53.3 \mathrm{MPa}$ if free ends and thus homogeneous strains are assumed. Actually, plasticity starts to occur in the middle 
of the shell on the outer wall, due to the built-in ends, at a lower pressure level. Nevertheless, without any defect or residual stress, bifurcation occurs in elasticity at the previous critical pressure $P_{c}$, even if plasticity is considered. In the post-buckling range, contrary to the elastic case, the critical point is followed by a limit point which is hardly above the bifurcation load. The maximum pressure $P_{\max }=39.9 \mathrm{MPa}$ defines the shell load carrying capacity and leads to unstable failure.

\subsubsection{Geometric imperfections}

Geometric imperfections are very often encountered in such cylindrical shells, due to the manufacturing process. In many cases, the inner and outer walls of the cylinder are not coaxial, what leads to heterogeneous strains and stresses in the shell. Moreover, the radius of the cylinder is known to be no longer constant, even if it is most often difficult to dispose of accurate measurements. In that study, geometric imperfections are taken into account in the form of an ovality of the initially perfect circular cylinder. This imperfection shape represents the prevalent and most damaging one (as far as buckling is concerned). The shell is supposed to have an elliptic shape and the defect amplitude is given by the radius difference $\Delta R=R_{\max }-R_{\min }$ between the two principal axes of the ellipse.

Next calculations are performed in order to show the influence of such an imperfection on the buckling and/or limit loads of the structure. Because precise measurements are not available, the imperfection amplitude is supposed to vary arbitrarily from $\Delta R=0$ to $0.4 \mathrm{~mm}$, in accordance with the dimensional tolerances of such extruded tubes.

Due to the built-in ends and most of all to this geometric imperfection, no bifurcation point is obtained, except for the smallest amplitude $\Delta R=0.05 \mathrm{~mm}$. Indeed, the buckling problem singularity is replaced by a degenerated regular equilibrium curve, just displaying large displacements and possibly limit points. Interesting results are thus naturally obtained when considering plasticity effects. Figure 13 depicts the maximum supported pressure versus the imperfection amplitude. It shows a non-negligible loss of the load carrying capacity of the shell when the ovality increases (the elastic buckling pressure is also added for comparison purposes).

\subsubsection{Residual stresses}

Finally, the axial and circumferential residual stress states presented above are included in the model in order to see the influence of initial stresses on the buckling behavior of a cylindrical shell under external pressure. 
First, purely elastic calculations are performed and allow us to conclude that residual stresses do not play any role in the elastic buckling of such shells. In the problem in hand, residual stresses will just increase more or less quickly the spreading plastic zone, due to non-homogeneous strains, and thus reduce the buckling stress until all the shell is plastified.

The results obtained in plasticity with residual stresses, for a perfect cylindrical shell or including the previous geometric imperfections of various amplitudes, are also plotted in Figure 13. Without any imperfection, the residual stresses seem to have no particular effect on the maximum pressure. This influence appears and grows with increasing imperfection amplitudes towards a constant value.

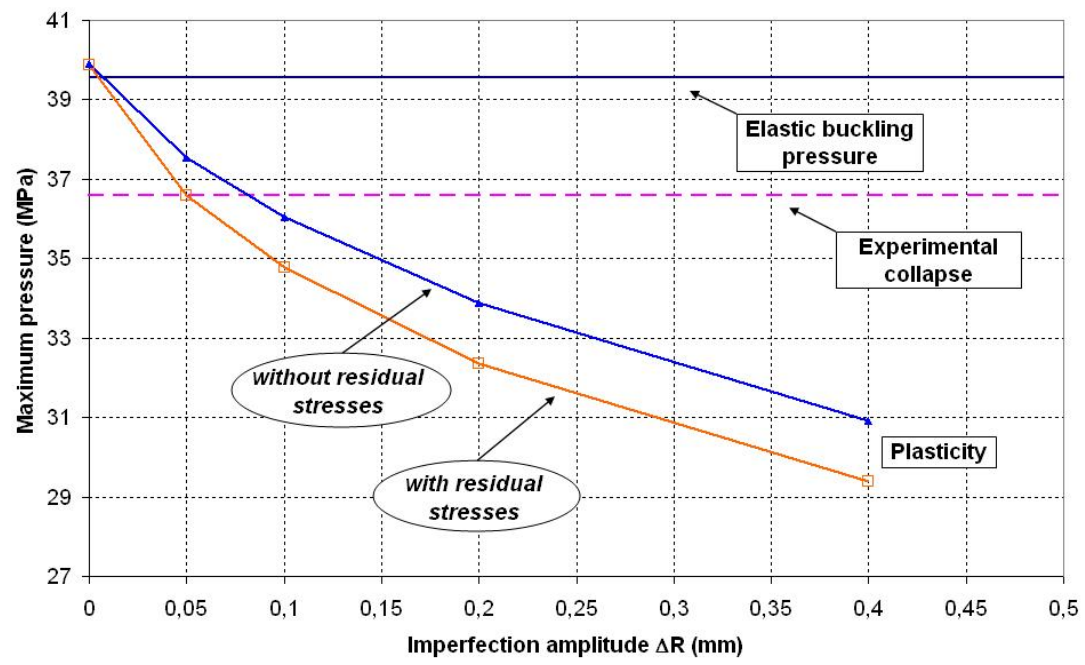

Fig. 13. Collapse external pressure of an elastoplastic cylindrical shell vs. geometric imperfection size, with or without residual stresses

\subsection{Validation and analysis}

First, the purely elastic and elastoplastic calculations have shown that elasticity and plasticity yielded totally different post-buckling behaviors in the case of a cylindrical shell under external pressure. In elasticity, the shell can support loadings above the critical pressure, even if large displacements are observed on the post-bifurcated equilibrium branch, what fatally leads to failure. On the contrary, when plasticity occurs in the vicinity of the buckling phenomenon, the bifurcation pressure is just followed by a maximum pressure on the post-critical curve, what clearly leads to an unstable collapse of the shell, as already observed by Dyau and Kyriakides (1993) and Kyriakides and Corona (2007), among others. Figures 14 and 15 show the applied external pressure versus the maximum radial displacement in the shell in elasticity and 
plasticity, respectively (the maximum imperfection amplitude $\Delta R=0.4 \mathrm{~mm}$ is considered, and residual stresses are included in plasticity).

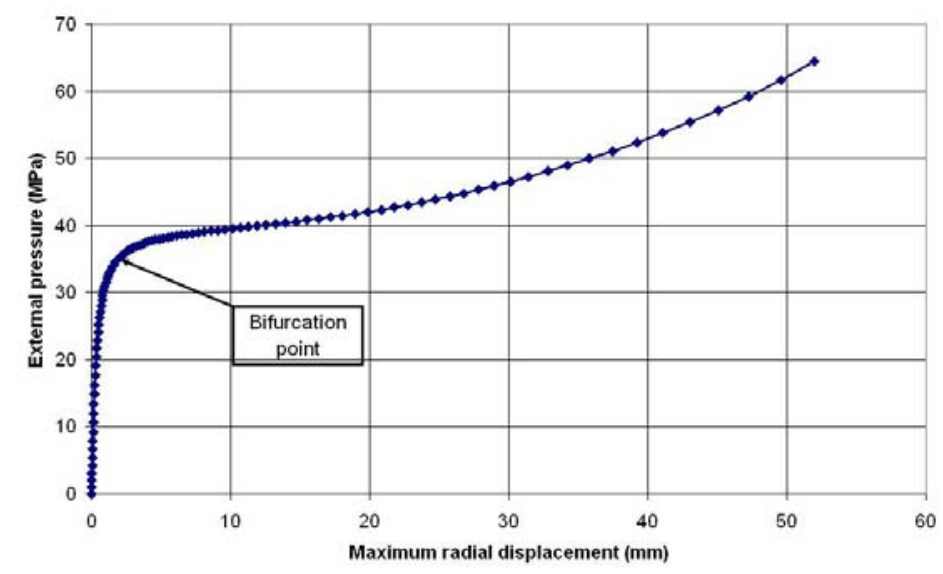

Fig. 14. External pressure vs. maximum radial displacement in the cylindrical tube: elasticity, ovality $\Delta R=0.4 \mathrm{~mm}$

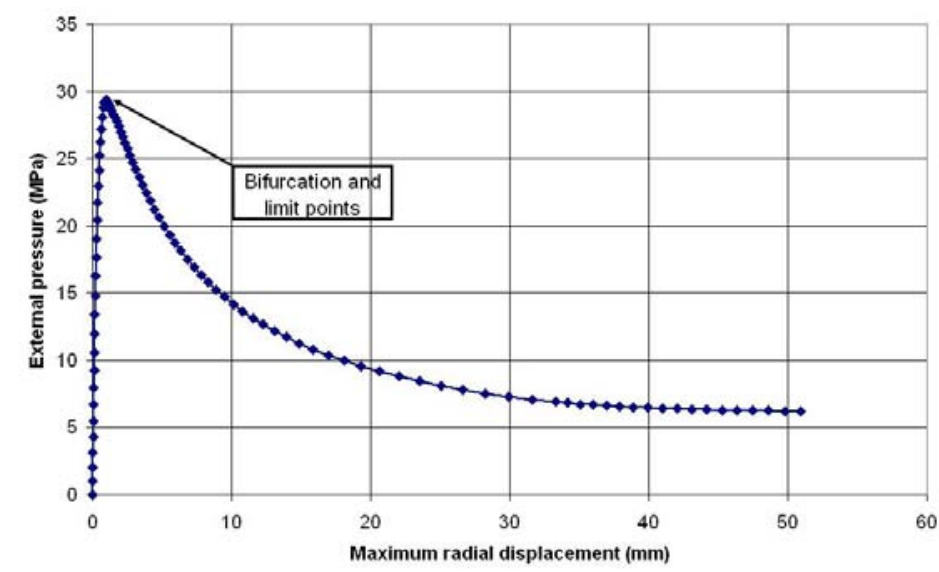

Fig. 15. External pressure vs. maximum radial displacement in the cylindrical tube: plasticity, ovality $\Delta R=0.4 \mathrm{~mm}$, residual stresses

Furthermore, the advanced deformed shape of the collapsed cylinder is depicted in Figures 16 and 17, in the elastic stable and plastic unstable cases, respectively. Despite identical buckling modes in both cases, the plasticity occurence, just localized in the middle of the shell, gives rise to the localization of buckling patterns at the same place, which is in better accordance with experimental results.

In a second stage, the imperfection sensitivity of such a shell has been illustrated, including some geometric imperfections (ovality of the initially circular shell) of various amplitudes. When the imperfection reaches the maximum value encountered in practice (only $0.75 \%$ of the average radius), the loss of carrying capacity attains $22.5 \%$ of the maximum pressure in the plastic perfect shell. 


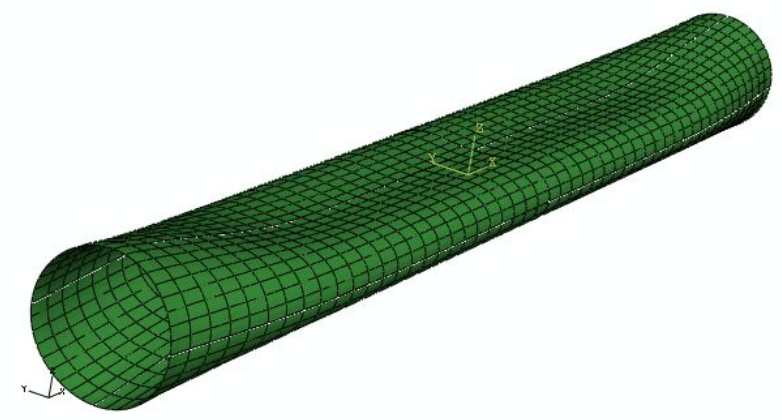

Fig. 16. Post-critical deformation shape of the cylindrical tube in the elastic case: maximum radial displacement $32.8 \mathrm{~mm}$, external pressure $48.1 \mathrm{MPa}$

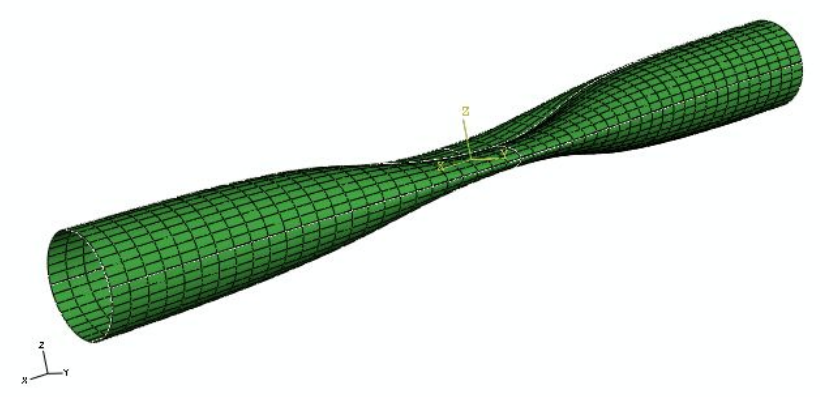

Fig. 17. Post-critical deformation shape of the cylindrical tube in the plastic case: maximum radial displacement $50.9 \mathrm{~mm}$, external pressure $6.19 \mathrm{MPa}$

Finally, residual stresses are considered in the model. The stronger influence of such initial manufacturing axial and particularly circumferential stresses occurs at the maximum imperfection amplitude. In the most detrimental case, the limit pressure does not exceed $75 \%$ of the elastic buckling pressure.

All the results show that neither plasticity effects, nor ovality and residual stresses can be neglected in such a buckling calculation. These three modeling components are really influential when considered together.

The experimental collapse load (366 bars) has also been plotted in Figure 13 for validation purposes. With the combined effects of plasticity, ovality and residual stresses, it is shown that, for the smallest imperfection amplitude considered $(\Delta R=0.05 \mathrm{~mm})$ which is inevitable in practice, the critical pressure, initially expected at a value of about $40 M P a$ perfectly coincides with the experimental value. 


\section{Conclusion}

In this work, a shell finite element model has been developed, encompassing finite rotations and plasticity, and involving appropriate arc-length and branching methods, in order to resolve the buckling and post-buckling of a cylindrical shell under combined axial and circumferential loading stresses. As mentioned by Schafer and Pekoz (1998), the primary impediment to success is neither the formulation nor the solution procedure but the lack of knowledge about the initial state, including residual stresses. Therefore, axial and circumferential residual stresses coming from the shell manufacturing process were accurately measured and included in the model. Geometric imperfections were also taken into account, considering the ovality of the initially perfect circular cylindrical shell.

Whereas plasticity and all imperfection types related to the initial state have a rather limited influence when considered separately, the combination of these several factors has a more significant effect. All these initial parameters, when carefully measured, enable us to enhance the numerical predictions of buckling or collapse pressure and thus improve the safety level of such structures.

\section{References}

Bardi, F.C. and Kyriakides, S., Plastic buckling of circular tubes under axial compression - part I: Experiments, International Journal of Mechanical Sciences 48 (2006) 830-841.

Bardi, F.C., Kyriakides, S. and Yun, H.D., Plastic buckling of circular tubes under axial compression - part II: Analysis, International Journal of Mechanical Sciences 48 (2006) 842-854.

Bergan, P.G., Horrigmoe, G., Krakeland, B. and Soreide, B., Solution techniques for non-linear finite element problem, International Journal for $\mathrm{Nu}$ merical Methods in Engineering 12 (1978) 1677-1696.

Brush, D.O. and Almroth, B.O., Buckling of Bars, Plates and Shells (Mc Graw-Hill, 1975).

Bushnell, D., Plastic buckling, Pressure Vessels and Piping: Design Technology - A Decade of Progress (S.Y. Zamrik and D. Dietrich, 1982) Book No. G00213 47-117.

Bushnell, D., Computerized Buckling Analysis of Shells (M. Nijhoff, 1985).

Corona, E. and Kyriakides, S., On the collapse of inelastic tubes under combined bending and pressure, International Journal of Solids and Structures 24 Issue 5 (1988) 505-535.

Corona, E. and Kyriakides, S., Asymmetric collapse modes of pipes under combined bending and external pressure, Journal of Engineering Mechanics 126 (2000) 1232-1239. 
Crisfield, M.A., Non-Linear Finite Element Analysis of Solids and Structures (Volume 1). Essentials (John Wiley \& Sons, 1991).

Dubey, R.N., Instabilities in thin elastic-plastic tubes, International Journal of Solids and Structures 5 Issue 7 (1969) 699-711.

Dyau, J.Y. and Kyriakides, S., On the localization of collapse in cylindrical shells under external pressure, International Journal of Solids and Structures 30 Issue 4 (1993) 463-482.

Green, A.E. and Naghdi, P.M., A general theory of an elastic-plastic continuum, Archive for Rational Mechanics and Analysis 18 (1965) 251-281.

Gusic, G., Combescure, A. and Jullien, J.F., The influence of circumferential thickness variations on the buckling of cylindrical shells under external pressure, Computers and Structures 74 (2000) 461-477.

Halphen, B. and Nguyen, Q.S., Sur les matériaux standard généralisés (On the generalized standard materials), Journal de Mécanique 14 Issue 1 (1975) 39-63.

Hill, R., A general theory of uniqueness and stability in elastic-plastic solids, Journal of the Mechanics and Physics of Solids 6 Issue 3 (1958) 236-249.

Hutchinson, J.W., Plastic buckling, Advances in Applied Mechanics 14 (1974) 67-144.

Jiao, H. and Zhao, X.L., Imperfection, residual stress and yield slenderness limit of very high strength (VHS) circular steel tubes, Journal of Constructional Steel Research 59 (2003) 233-249.

Kheyrkhahan, M. and Peek, R., Post-buckling analysis and imperfection sensitivity of general shells by the finite element method, International Journal of Solids and Structures 36 Issue 18 (1999) 2641-2681.

Kyriakides, S. and Corona, E., Mechanics of Offshore Pipelines. Volume 1: Buckling and Collapse (Elsevier, 2007).

Lam, W.F. and Morley, C.T., Arc-length method for passing limit points in structural calculation, Journal of Structural Engineering 118 Issue 1 (1992) $169-185$.

Lancaster, E.R., Calladine, C.R. and Palmer, S.C., Paradoxical buckling behavior of a thin cylindrical shell under axial compression, International Journal of Mechanical Sciences 42 (2000) 843-865.

Le Grognec, P. and Le van, A., Elastoplastic bifurcation and collapse of axially loaded cylindrical shells, International Journal of Solids and Structures $\mathbf{4 5}$ Issue 1 (2008) 64-86.

Mandal, P. and Calladine, C.R., Buckling of thin cylindrical shells under axial compression, International Journal of Solids and Structures 37 Issue 33 (2000) 4509-4525.

Riks, E., An incremental approach to the solution of snapping and buckling problems, International Journal of Solids and Structures 15 Issue 7 (1979) 529-551.

Riks, E., On formulations on path-following techniques for structural stability analysis, New Advances in Computational Structural Mechanics (Elsevier, 1991) 65-80. 
Schafer, B.W. and Pekoz, T., Computational modeling of cold-formed steel: characterizing geometric imperfections and residual stresses, Journal of Constructional Steel Research 47 (1998) 193-210.

Seydel, R., Practical Bifurcation and Stability Analysis. From Equilibrium to Chaos (Springer-Verlag, 1994).

Shanley, F.R., Inelastic column theory, International Journal of Aeronautical Sciences 14 (1947) 261-267.

Simo, J.C. and Hughes, T.J.R., Computational Inelasticity (Springer, 1998).

Singer, J., On the importance of shell buckling experiments, Applied Mechanics Review 52 Issue 6 (1999).

Timoshenko, S.P. and Gere, J.M., Theory of Elastic Stability (Mc Graw-Hill, 1961).

Triantafyllidis, N., On the bifurcation and post-bifurcation analysis of elasticplastic solids under general pre-bifurcation conditions, Journal of the Mechanics and Physics of Solids 31 Issue 6 (1983) 499-510.

Tvergaard, V., Plastic buckling of axially compressed circular cylindrical shells, Thin-Walled Structures 1 Issue 2 (1983) 139-163.

Wagner, W. and Wriggers, P., A simple method for the calculation of postcritical branches, Engineering and Computations 5 (1988) 103-109.

Yeh, M.K. and Kyriakides, S., On the collapse of inelastic thick-walled tubes under external pressure, Journal of Energy Resources Technology 108 (1986) 35-47.

Yeh, M.K. and Kyriakides, S., Collapse of deepwater pipelines, Journal of Energy Resources Technology 110 (1988) 1-11. 